\title{
COMMENTS
}

Comments are short papers which criticize or correct papers of other authors previously published in Physical Review B. Each Comment should state clearly to which paper it refers and must be accompanied by a brief abstract. The same publication schedule as for regular articles is followed, and page proofs are sent to authors.

\section{Comment on "Periodic wave functions and number of extended states in random dimer systems",}

\author{
M. Hilke \\ Department of Electrical Engineering, Princeton University, Princeton, New Jersey 08544 \\ J. C. Flores \\ Departamento de Física, Universidad de Tarapacá, Casilla 7-D, Arica, Chile \\ F. Domínguez-Adame \\ Departamento de Física de Materiales, Universidad Complutense, E-28040 Madrid, Spain
}

(Received 12 November 1997)

\begin{abstract}
In a recent report, Huang, Wu, and Gong [Phys. Rev. B 55, 11018 (1997)] studied numerically the electronic properties of a random dimer model (RDM) and found extended periodic wave functions near the critical energies. We argue that there are no periodic wave functions in the RDM but close to the critical energies there exist periodic envelopes. These envelopes are given by the nondisordered properties of the system. [S0163-1829(98)10337-5]
\end{abstract}

In a recent paper, ${ }^{1}$ Huang, $\mathrm{Wu}$, and Gong studied numerically the electronic properties of a random dimer model (RDM) and found extended periodic wave functions near the critical energies. They also claimed that the number of extended states is proportional to $\sqrt{N}$, where $N$ is the size of the system, which is consistent with previous results.

(i) There are no periodic wave functions in the RDM. Indeed, even at the critical energies it was shown previously ${ }^{2}$ that the wave functions have a constant norm but the phases are random. Essentially the wave function is $\psi_{i}=e^{i \theta_{i}}$, where $\theta_{i}$ is random. This does, however, lead to a constant envelope. We define the envelope as the ensemble of curves which include all possible values of the random wave function. In this point we present the analytical derivation against the main result presented in Ref. 1 and show that the envelope (and not the wave function itself) is periodic near the critical energies as long as the localization length exceeds the size of the system.

The RDM can be analyzed in terms of the product of the following two transfer matrices ${ }^{3}$

$$
T_{A}=\left(\begin{array}{cc}
E-V_{A} & -1 \\
1 & 0
\end{array}\right)^{2}, \quad T_{B}=\left(\begin{array}{cc}
E-V_{B} & -1 \\
1 & 0
\end{array}\right)^{2} .
$$

They reduce to the negative identity matrix when $E=V_{A}$ or $E=V_{B}$ (critical energies), which means that $T_{A}$ and $T_{B}$ commute. Close to the critical energy, i.e., when $\Delta E=E-V_{A}$ is small, the commutator $\left[T_{A}, T_{B}\right]=O(\Delta E)$, as opposed to the case without the dimer condition, where the commutator is always of order 1 . The total system is described by a random mixture of the products of the transfer matrices $T_{A}$ and $T_{B}$, thus $T=\Pi_{i} T_{A}^{n_{i}} T_{B}^{m_{i}}$, where $n_{i}$ and $m_{i}$ are random. Therefore the total product is $T=T_{A}^{n} T_{B}^{m}+(N / 4) O(\Delta E)$, where $n$ $=\sum_{i} n_{i}, m=\sum_{i} m_{i}$, and $N=2(n+m)$. This clearly demonstrates that the solution is equivalent to the system with

$$
T=(-1)^{n} T_{B}^{m},
$$

as long as $N$ is smaller than $1 / \Delta E$.

Our main result is that the envelope of Eq. (2) reproduces all the numerical figures of Ref. 1. Indeed Huang, $\mathrm{Wu}$, and Gong studied numerically two cases:

(a) $V_{A}=-1, V_{B}=1$, and $E=-1+\Delta E$. As the envelope can be obtained from the solution of $T_{B}^{m}$, the corresponding wave functions can be written as $\psi_{i} \sim \cos (k i)$, where $E-V_{B}$ $=2 \cos (k)$. To first order in $\Delta E$ we obtain $k \simeq \pi-\sqrt{\Delta E / 2}$. From this follows trivially that the general solution has periodic solutions for $2 m \sqrt{\Delta E / 2}=\pi p$, where $p$ is an integer. This leads to $\Delta E=2 \pi^{2} p^{2} /(N / 2)^{2}$, where $N / 2$ is the number of dimers when they are equally distributed.

(b) $V_{A}=-0.5, V_{B}=0.5$, and $E-V_{B}=-1+\Delta E$. In this case we obtain $k \simeq 2 \pi / 3-\sqrt{3} \Delta E / 2$ and $\Delta E=2 \pi p /$ $\sqrt{3}(N / 2)$.

In both cases (a) and (b) the same dependences as in Ref. 1 are obtained.

(ii) The use of the inverse participation ratio (IPR) IPR $=N \Sigma_{j=1}^{N}\left|\psi_{j}\right|^{4}$, where $\psi_{j}$ is the normalized wave function at site $j$, is very ambiguous. We can, indeed, construct delocalized wave functions with arbitrary IPR. For instance, when considering the following periodic sparse and normalized 
wave function $\psi_{j}^{2}=T / N$ if $j / T=$ integer and $\psi_{j}^{2}=0$ otherwise, where the period $T$ is an integer $(T<N)$, then IPR $=T$. In this way we have a set of delocalized states with arbitrary IPR. Therefore, the relevant quantity to study is the scaling of IPR with $N$, which gives consistent results even for sparse states as, for example, in banded random matrices. ${ }^{4}$ For a more general approach a multifractal analysis is proved useful. ${ }^{5}$

(iii) The number of extended states is proportional to $\sqrt{N}$, which means that the relative number of extended states tends to zero as $1 / \sqrt{N}$; therefore the delocalization properties are important only in small systems. ${ }^{6}$

In conclusion, there are no periodic wave functions in the RDM, but close to the critical energies there exist periodic envelopes. These envelopes are given by the nondisordered properties of the system.

Part of this work was made possible due to the project FDI-Chile (J.C.F. and M.H.).
${ }^{1}$ X. Huang, X. Wu, and C. Gong, Phys. Rev. B 55, 11018 (1997).

${ }^{2}$ J. C. Flores and M. Hilke, J. Phys. A 26, L1255 (1993).

${ }^{3}$ M. Hilke, J. Phys. A 27, 4773 (1994).

${ }^{4} \mathrm{Ph}$. Jacquod and D. L. Shepelyansky, Phys. Rev. Lett. 75,
3501 (1995).

${ }^{5}$ F. Domínguez-Adame, E. Maciá, and A. Sánchez, Phys. Rev. B 48, 6054 (1993).

${ }^{6}$ M. Hilke and J. C. Flores, Phys. Rev. B 55, 10625 (1997). 